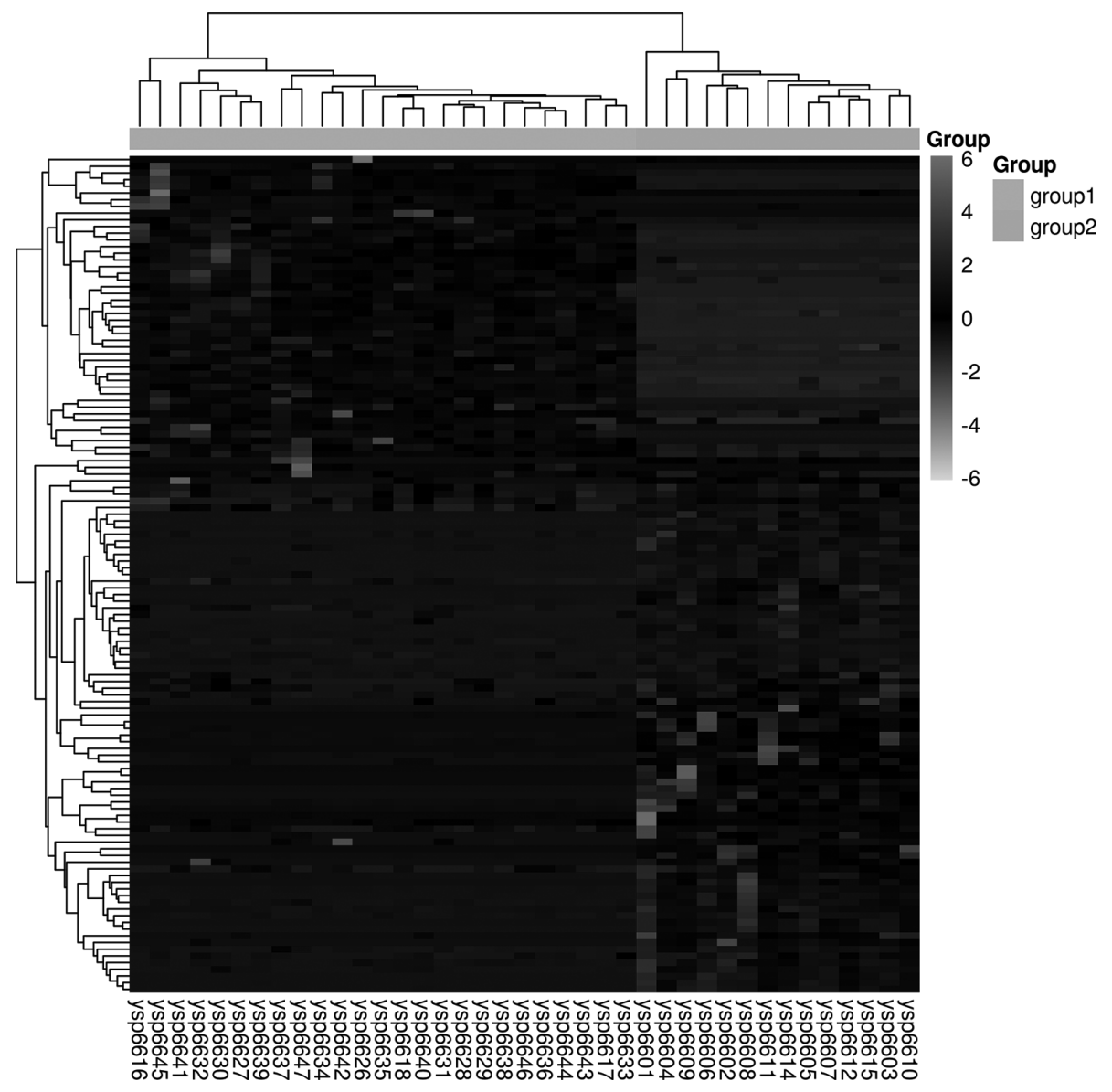

Abstract IDDF2018-ABS-0087 Figure 2

\section{IDDF2018-ABS-0089 WHO IS THE CHIEF CULPRIT OF THE ULCER?}

HuiJuan Liu*. The General Hospital of Ningxia Medical University, China

\subsection{6/gutjnl-2018-IDDFabstracts. 103}

Background As a painkiller, NSAID is widely used in the treatment of rheumatoid arthritis, osteoarthritis and other rheumatic diseases. Its common side effects are mainly lead to gastrointestinal damage, kidney damage, liver and blood other system damage. NSAIDs related gastrointestinal disease is not uncommon, which mostly occur in the stomach and duodenum, and it is extremely rare in the large intestine, only a few cases have been found in this location to date.

Methods A 66-year-old man who presented abdominal pain and hematochezia, was admitted to our hospital in May 2017. The pain was located in the umbilicus and lower abdomen, and hematochezia 6-7 times a day. These symptoms lasted about 20 days. No abnormal finding was revealed throughout physical examination except slight tenderness around the cord and lower abdomen. Gastroscopy found a gastric ulcer and Colonoscopy found multiple ulcers in the colon (figure 1a, 2a). What are these ulcers? Do gastric ulcer and colon ulcer have the same aetiology, or they have their own aetiology? So laboratory tests (T-spot, CEA, CA19-9, Related rheumatology indicators, pathology) and image examinations (Abdominal Bultra, CT) were taken. But these examinations cannot fully support the diagnosis of similar symptoms, such as inflammation, cancer, tuberculosis, and ischaemic bowel disease. We asked the patient history carefully again. He told us that he took a few tablets of NSAID to control neck and shoulder pain more than 4 years. We guessed that he was NSAIDsrelated gastrointestinal disease.

Results So we suggested the patient stop taking NSAIDs, and give omeprazole, mucosa protectant and probiotics to treat these ulcers. Two months later, gastroscopy and colonoscopy found that ulcers healed (figure $1 \mathrm{~b}, 2 \mathrm{~b}$ ).

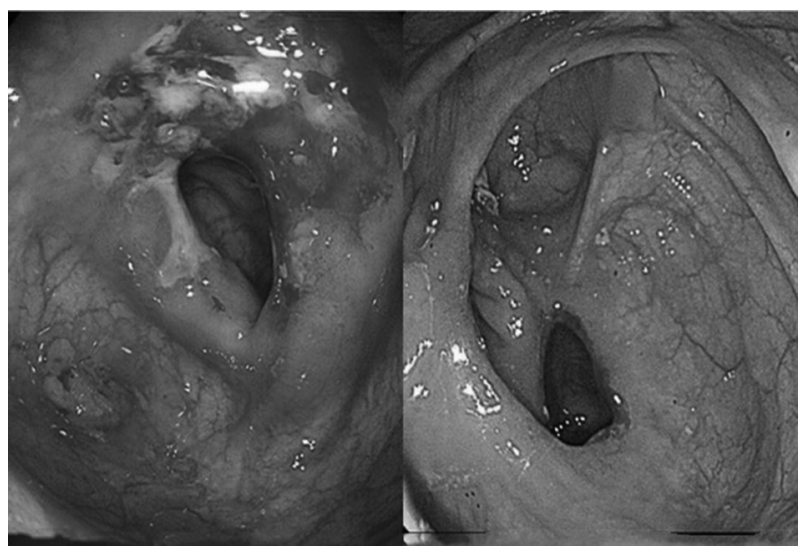

Abstract IDDF2018-ABS-0089 Figure 1 (a) Colonoscopy and gasreoscopy images at different times; the colon ulcer May 18, 2017; (b) Colonoscopy and gasreoscopy images at different times; stop taking NSAIDs after two months, the healed ulcer July 24, 2017 


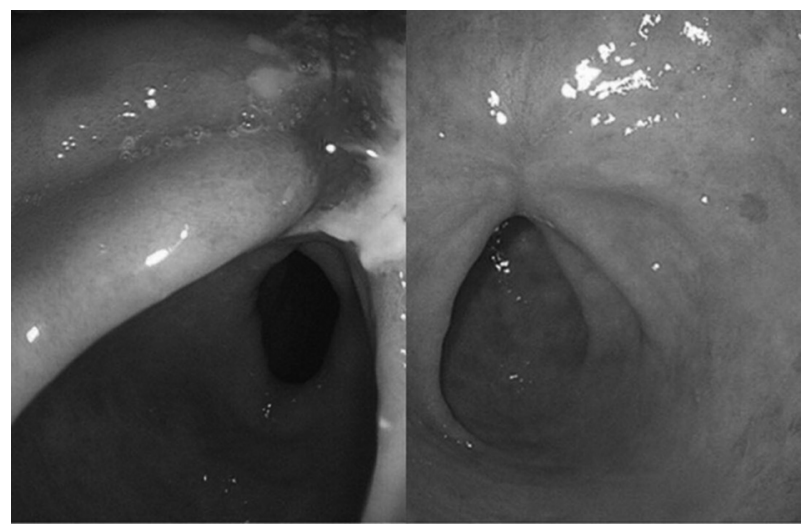

Abstract IDDF2018-ABS-0089 Figure 2 (a) Colonoscopy and gasreoscopy images at different times; the gastric ulcer May 19, 2017; (b) Colonoscopy and gasreoscopy images at different times; stop taking NSAIDs after two months, the healed ulcer July 24, 2017

Conclusions These results were shown NSAIDs were the chief culprit of the ulcer. We should alert the intestinal mucosal injury of NSAIDs.

\section{IDDF2018-ABS-0090 BILATERAL ADRENALECTOMY AND SEGMENT VI RESECTION OF LIVER IN A YOUNG MAN WITH VON HIPPEL-LINDAU DISEASE}

Nitin Agarwal*, Rana Anil Kumar Singh, Peeyush Kumar. Department of Surgery and Renal Transplant Postgraduate Institute of Medical Education and Research (PGIMER) and Dr Ram ManohaLohia Hospital, Baba Kharak Singh Marg, New Delhi, India

\subsection{6/gutjnl-2018-IDDFabstracts. 104}

Background Bilateral pheochromocytomas can be seen in von Hippel-Lindau disease (VHL) and Multiple Endocrine Neoplasia (MEN) syndrome. Metastases occur in 10\%-15\% of all pheochromocytomas. Resection of the primary, metastasectomy and/or debulking are acceptable surgical options; despite liver being the second most common site of metastases, only a few reports of concomitant adrenalectomy and liver resection for phaeochromocytoma are available.

Methods A 33-year-old man with a history of excision of hemangioblastoma of the cauda equina and retinal detachment presented with secondary hypertension and palpitations. Urinary catecholamine levels were raised, and CT scan revealed $\sim 4.5 \mathrm{~cm}$-sized, well-defined heterogeneously-enhancing lesions in bilateral adrenals with a $6.5 \times 5 \mathrm{~cm}$ lesion in segment VI of the liver. MRI showed heterogeneous signal intensity in bilateral adrenals (predominantly hypointense on T1W and hyperintense on T2W) and also in segment VI of the liver with loss of fat planes. ${ }^{131}$-MIBG scan with SPECT/SPECTCT showed uptake of tracer in the area of the bilateral adrenal masses and liver. With a diagnosis of VHL disease with bilateral phaeochromocytomas, recurrent/residual spinal hemangioblastoma, right simple renal cyst, retinal detachment and a metastatic/primary liver lesion, the patient was explored, and bilateral adrenalectomy with segmentectomy VI was performed.

Results The resection was performed with curative intent; histopathological examination revealed malignant phaeochromocytoma with infiltrates in the liver. Resection margins were negative and the patient is normotensive six months postoperatively. Though bilateral phaeochromocytomas are well-known in many familial syndromes, this is probably the first case of VHL to have undergone bilateral adrenalectomy and curative liver resection for metastatic phaeochromocytoma in the available English-language literature. Hepatectomy for neuroendocrine tumours especially phaeochromocytoma can be technically easier due to the well-circumscribed nature of the lesion, and also rewarding as complete removal leads to sudden relief from symptoms of hormone excess. Due to favourable histopathology in our patient, we are hopeful for a good long-term survival.

Conclusions Familial bilateral phaeochromocytomas have a high propensity for malignancy. Surgical resection should be attempted for metastatic phaeochromocytoma whenever possible, i.e., with limited metastatic disease, good performance status, and in an equipped centre.

\section{IDDF2018-ABS-0091 FOLK HERBAL MEDICINE FOR ORAL ULCER: A QUALITATIVE INTERVIEW WITH 20 THAI FOLK HEALERS}

${ }^{1}$ Siraporn Mahakoat ${ }^{*},{ }^{1}$ Sujaree Panomket, ${ }^{2}$ Bunleu Sungthong, ${ }^{3}$ Wikit Prakaiharn, ${ }^{4}$ Krit Pongpirul. ${ }^{1}$ Kamalasai Hospital, Thailand; ${ }^{2}$ Mahasarakham University, Thailand; ${ }^{3}$ Ministry of Public Health, Thailand; ${ }^{4}$ Chulalongkorn University, Thailand

\subsection{6/gutjnl-2018-IDDFabstracts. 105}

Background Uncomplicated oral ulcer is common in both adult and children. In addition to symptomatic treatment using western medicine, many traditional herbal recipes for oral ulcer have been less recognised and should be preserved. This study aimed to explore existing folk medicine knowledge and herbal recipes.

Methods This qualitative study applied both semi-structured interviews with 20 purposively selected folk healers in Kalasin, Thailand and ethnobotany methodology. The interviews focused on oral ulcer diagnosis and treatment experience as well as essential details of the ingredients. The data were analysed using thematic content analysis and descriptive statistics.

Results Five herbal recipes and 17 herbs (28 species from 16 families) were identified from the interview. Nine species from family FABACEAE (LEGUMINASAE) were the most commonly mentioned for their astringent and anti-inflammatory effects. Regarding the part used of medicinal plants, the survey indicated that the use of barks, from 10 species, were frequently found. With informant consensus factor, Areca catechu Linn. and Sesbania grandiflora (Desv.) Linn. were the most recognised by 12 and 11 folk healers, respectively.

Conclusions Herbal species from the family FABACEAE have been considered useful by Thai folk healers in Kalasin. Their astringent and anti-inflammatory properties should be further tested. 